DOI: $10.17516 / 1997-1397-2021-14-1-74-86$

УДК $532.711+66.081 .6$

\title{
Modelling the Ionic Conductivity of Nanopores with Electrically Conductive Surface
}

\author{
Artur I. Krom* \\ Institute of Computational Modelling of SB RAS \\ Krasnoyarsk, Russian Federation \\ Maria I. Medvedeva ${ }^{\dagger}$ \\ Siberian Federal University \\ Krasnoyarsk, Russian Federation \\ Ilya I. Ryzhkov \\ Institute of Computational Modelling of SB RAS \\ Krasnoyarsk, Russian Federation \\ Siberian Federal University \\ Krasnoyarsk, Russian Federation
}

Received 18.07.2020, received in revised form 25.09.2020, accepted 05.11.2020

\begin{abstract}
The ionic conductivity of nanopores with electrically conductive surface is investigated theoretically. The generalization of two-dimensional (2D) Space-charge model to calculating the ion transport under the applied potential gradient in a nanopore with constant surface potential is proposed for the first time. The results are compared with one-dimensional (1D) Uniform potential model, which is derived from the Space-charge model by assuming the independence of potential, ion concentrations, and pressure on the radial coordinate. We have found that the increase of surface potential magnitude leads to the enhancement of conductivity due to the increase of counter-ion concentration inside the nanopore. It is shown that the $1 \mathrm{D}$ and $2 \mathrm{D}$ models provide close results when the pore radius is smaller than the Debye length. Otherwise, the 1D model essentially overestimates the ionic conductivity. According to the $2 \mathrm{D}$ model, the ionic conductivity decreases with increasing the nanopore radius, while the $1 \mathrm{D}$ model predicts the opposite trend, which is not physically correct.
\end{abstract}

Keywords: nanopore, ionic conductivity, Space charge model, Uniform potential model.

Citation: A.I. Krom, M.I. Medvedeva, I.I. Ryzhkov, Modelling the Ionic Conductivity of Nanopores with Electrically Conductive Surface, J. Sib. Fed. Univ. Math. Phys., 2021, 14(1), 74-86.

DOI: 10.17516/1997-1397-2021-14-1-74-86.

\section{Introduction}

The ion transport in single nanochannels and nanoporous membranes is relevant in many technological applications such as separation and purification [1], water treatment [2], energy harvesting and conversion [3], electrochemical sensors [4], and nanofluidic devices [5]. When ionic solutions are confined in nanoscale geometries, the interactions of ions with the surface become of primary importance. The transport of ions can be switched or tuned by combining the variation of pore geometry and surface physicochemical properties with external stimuli, such as electric field, radiation, temperature, solution $\mathrm{pH}$, etc. [6, 7].

\footnotetext{
*kromarthur@gmail.ru

$\dagger$ mimedvedeva@rambler.ru

${ }^{\ddagger}$ rii@icm.krasn.ru

(c) Siberian Federal University. All rights reserved
} 
The ability of nanoporous media to transport ions under the applied electric field is characterized by the ionic conductivity, which is the ratio of ionic current to the voltage difference. Membranes with high conductivity are advantages in such processes as electrodialysis [8], capacitive deionization [9], and hydrogen energy conversion [10]. The experimental measurements of ionic conductivity, streaming potential, and potential at zero current in mica membranes were performed in [11]. The conductivity of ion-exchange membranes under alternating current was investigated in [12]. Recently, the measurements of ionic current through microfluidic devices containing one or several single wall carbon nanotubes revealed linear or voltage-activated current-voltage dependence [13]. Nanoporous membranes with electrically conductive surface were suggested in [14] by electroless plating of gold on the pore walls of track-etched polymeric support. It was shown that their ionic selectivity can be switched from cation to anion by varying the potential applied to the membrane surface. The authors of [15] found that the increase of applied potential magnitude resulted in the enhancement of ionic conductivity in gold nanotube membranes. The effect of changing the ionic selectivity and conductivity of alumina nanofiber membranes with conductive carbon coating was demonstrated in [16].

The theoretical description of ion transport in charged nanoporous membranes was developed in [17] on the basis of Navier-Stokes, Nernst-Planck, and Poisson equations. Now it is known as the two-dimensional Space-charge (SC) model, which takes into account the radial and longitudinal variations of potential, ion concentrations, and pressure in a cylindrical nanopore with constant surface charge density. This model was successfully employed for predicting the ionic conductivity in mica and alumina membranes $[11,18]$. The SC model was revisited recently [19] by providing an essential simplification of working formulas, and further used to predict the ionic conductivity of carbon nanotubes [20]. The extension of SC model to the nanopores with constant surface potential was first suggested in [22] and applied to description of membrane potential at zero current in conductive nanopores [23-25]. It was demonstrated that the induced charge effects can essentially affect the magnitude of membrane potential. The simplification of SC model known as Uniform potential (UP) model was suggested in [19] by neglecting the radial variations of potential, ion concentrations, and pressure. This one-dimensional model is valid for nanopores, which effective diameter is comparable with or smaller than the Debye length. The extension of UP model to the presence of both electronic and $\mathrm{pH}$-dependent chemical charges was proposed in [26]. It was successfully employed in [27] for describing the experimental results of [14] on switchable ionic selectivity. It should be noted that the studies of carbon nanotube [13] and gold nanotube $[14,15]$ membranes rise interest in the theoretical analysis of ionic conductivity on the basis of SC and UP models with constant surface potential boundary condition. The comparative analysis of these models could provide the conditions, under which the two-dimensional $\mathrm{SC}$ model can be replaced by one-dimensional UP model with much lower computational cost.

In this work, we theoretically investigate the ionic conductivity of nanopores with electrically conductive surface and its dependence on the surface potential and nanopore radius. The comparison between results obtained from two-dimensional SC model and one-dimensional UP model is performed.

\section{Mathematical models of nanopore ionic conductivity}

\subsection{Two-dimensional space charge model}

We are interested in studying the ionic current through a nanoporous membrane, which separates two reservoirs denoted by $L$ (left) and $R$ (right). The reservoirs contain aqueous 
solutions of the same monovalent and symmetric (1:1) electrolyte with concentration $C_{0}$. The motion of ions is induced by the electric field, which is imposed by specifying different potentials $\Phi_{L}$ and $\Phi_{R}$ in the reservoirs. The pressures in the reservoirs are equal and taken to be zero for simplicity. The potential $\Phi_{s}$ is specified on the electrically conductive membrane surface.

A membrane is modelled as an array of cylindrical pores of length $L_{p}$ and radius $R_{p}$. Thus, it is enough to calculate the ion transport in a single pore, where the cylindrical coordinates $R$ in radial and $Z$ in axial directions are introduced (Fig. 1). The transport of electrolyte through the pore is characterized by the solution velocity $\boldsymbol{U}=(U, V)$, pressure $P$, cation $C_{+}$ and anion $C_{-}$concentrations $\left(\mathrm{mol} / \mathrm{m}^{3}\right)$, and electric potential $\Phi$, which are functions of axial and radial coordinates. These quantities satisfy the system of two-dimensional Navier-Stokes, Nernst-Planck, and Poisson equations [11, 19,23].

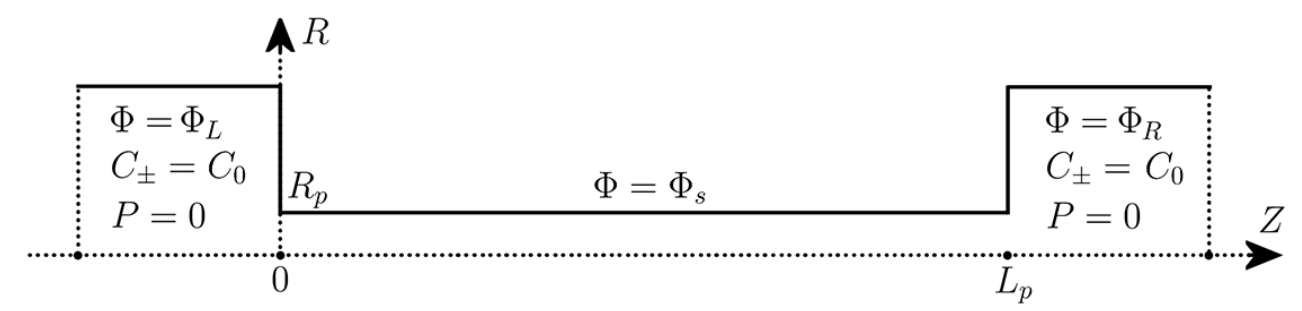

Fig. 1. The geometry of a single cylindrical pore

The ions in the nanopore are transported by convection, diffusion, and migration in electric field. The fluxes of ions are written as

$$
\boldsymbol{J}_{ \pm}=C_{ \pm} \boldsymbol{U}-D_{ \pm} \nabla C_{ \pm} \mp \frac{D_{ \pm} F}{R_{g} T} C_{ \pm} \nabla \Phi
$$

where $D_{ \pm}$are the ion diffusion coefficients, $R_{g}$ is the ideal gas constant, $T$ is the temperature, and $F$ is the Faraday constant.

Let us introduce dimensionless variables by

$$
\begin{gathered}
R=R_{p} r, \quad Z=L_{p} z, \quad \boldsymbol{U}=\frac{D_{+}}{L_{p}} \boldsymbol{u}, \quad P=C_{*} R_{g} T p \\
C_{ \pm}=C_{*} c_{ \pm}, \quad \Phi=\frac{R_{g} T}{F} \varphi, \quad \boldsymbol{J}_{ \pm}=\frac{D_{+} C_{*}}{L_{p}} \boldsymbol{j}_{ \pm} \cdot
\end{gathered}
$$

Here $\boldsymbol{u}=(u, v)$ and $C_{*}$ is the reference concentration taken as $C_{*}=1 \mathrm{~mol} / \mathrm{m}^{3}$.

In what follows, we will need the quantities averaged over the pore cross-section. The dimensional average axial velocity is defined by

$$
\bar{V}=\frac{2}{R_{p}^{2}} \int_{0}^{R_{p}} V R d R .
$$

The average pressure $\bar{P}$, ion concentrations $\bar{C}_{ \pm}$, potential $\bar{\Phi}$, axial ion fluxes $\bar{J}_{ \pm}$, total axial ion flux $\bar{J}=\bar{J}_{+}+\bar{J}_{-}$, and axial charge flux $\bar{I}=\bar{J}_{+}-\bar{J}_{-}$are introduced in the same way. The corresponding dimensionless quantities are $\bar{v}, \bar{p}, \bar{c}_{ \pm}, \bar{\varphi}, \bar{j}_{ \pm}, \bar{j}=\bar{j}_{+}+\bar{j}_{-}$, and $\bar{i}=\bar{j}_{+}-\bar{j}_{-}$.

The ionic conductivity $G(\mathrm{~S})$ is the ratio between the ionic current $I$ and the potential difference $U$ :

$$
G=\frac{I}{U}
$$


For the nanopore shown in Fig. 1, one should put $U=\Phi_{L}-\Phi_{R}$, while the ionic current through the pore is given by $I=\pi R_{p}^{2} F \bar{I}$. Thus, a positive voltage drop between left and right reservoirs induces positive current, i.e. positive charge is transported in the direction of electric field. The specific conductivity $\kappa(\mathrm{S} / \mathrm{m})$ of a cylindrical nanopore is defined by

$$
\kappa=G \frac{L_{p}}{\pi R_{p}^{2}}
$$

Let us express it through the dimensionless average charge flux and potential difference:

$$
\kappa=\frac{D_{+} C_{*} F^{2}}{R_{g} T} \frac{\bar{i}}{\varphi_{L}-\varphi_{R}} .
$$

We use the two-dimensional Space-Charge (SC) model for describing the transport of ions through the nanopore and calculating the ionic current and conductivity. The SC model is derived from the Navier-Stokes, Nernst-Planck, and Poisson equations by introducing several assumptions appropriate for large aspect ratio pores [19]. The dimensionless potential $\varphi$, ion concentrations $c_{ \pm}$, and pressure $p$ are represented as

$$
\begin{gathered}
\varphi(r, z)=\phi_{v}(z)+\psi(r, z), \quad c_{ \pm}(r, z)=c_{v}(z) \exp (\mp \psi(r, z)) \\
p(r, z)=p_{v}(z)+2 c_{v}(z) \cosh (\psi(r, z))
\end{gathered}
$$

Here the ion concentrations satisfy the Boltzmann distribution. The function $\psi$ satisfies the Poisson equation with the boundary condition of axial symmetry

$$
\begin{gathered}
\frac{1}{r} \frac{\partial}{\partial r}\left(r \frac{\partial \psi(r, z)}{\partial r}\right)=\frac{c_{v}(z)}{\lambda^{2}} \sinh \psi(r, z), \\
\frac{\partial \psi}{\partial r}(0, z)=0 .
\end{gathered}
$$

Here $\lambda=\sqrt{\varepsilon \varepsilon_{0} R_{g} T / 2 F^{2} C_{*}} / R_{p}$ is the dimensionless Debye length, $\varepsilon$ is the relative permittivity, and $\varepsilon_{0}$ is the dielectric constant. At $r=1$, we impose the condition

$$
\psi(1, z)=\varphi_{s}-\phi_{v}(z)
$$

which is derived from (4) by assuming the constant potential on the nanopore surface. The generalization of Space-Charge model to constant surface potential case was first proposed in [22].

The relation of the average volume flux $\bar{v}$ (or average axial velocity), average ion flux $\bar{j}=\bar{j}_{+}+\bar{j}_{-}$, and average charge flux $\bar{i}=\bar{j}_{+}-\bar{j}_{-}$to the gradients of virtual pressure $p_{v}$, virtual chemical potential $\mu_{v}=\ln c_{v}$, and virtual electric potential $\phi_{v}$ can be written in the form of phenomenological flux-force formalism [19]:

$$
\left(\frac{d p_{v}}{d z}, \frac{d \mu_{v}}{d z}, \frac{d \phi_{v}}{d z}\right)^{T}=L(\bar{v}, \bar{j}, \bar{i})^{T}
$$

Here $L=-\mathcal{L}^{-1}$ is the symmetric $3 \times 3$ matrix. The coefficients of matrix $\mathcal{L}=\left\{\mathcal{L}_{i j}(z)\right\}$ depend 
on the function $\psi(r, z)$ and virtual concentration $c_{v}(z)$ according to $[19,23]$

$$
\begin{gathered}
\mathcal{L}_{11}=\frac{1}{8 \alpha}, \quad \mathcal{L}_{22}=2 c_{v} \int_{0}^{1} r(D \exp (\psi(r))+\exp (-\psi(r))) d r- \\
-\frac{16 c_{v}^{2}}{\alpha} \int_{0}^{1}\left[r \cosh (\psi(r)) \ln r\left(\frac{r^{2}}{2} \cosh (\psi(r))-\frac{\lambda^{2}}{4 c_{v}}\left(r \frac{\partial \psi(r)}{\partial r}\right)^{2}\right)\right] d r, \\
\mathcal{L}_{33}=-\frac{8 c_{v}}{\alpha} \int_{0}^{1} r\left[\sinh (\psi(r)) \lambda^{2}\left(\psi(r)-\psi_{s}\right)-\frac{\alpha}{4}(D \exp (\psi(r))+\exp (-\psi(r)))\right] d r, \\
\mathcal{L}_{12}=\mathcal{L}_{21}=\frac{c_{v}}{\alpha} \int_{0}^{1}\left(r-r^{3}\right) \cosh (\psi(r)) d r, \quad \mathcal{L}_{13}=\mathcal{L}_{31}=\frac{4}{\alpha} \int_{0}^{1} r \lambda^{2}\left(\psi(r)-\psi_{s}\right) d r, \\
\mathcal{L}_{23}=\mathcal{L}_{32}=\frac{8 c_{v}}{\alpha} \int_{0}^{1} r\left[\cosh (\psi(r)) \lambda^{2}\left(\psi(r)-\psi_{s}\right)-\frac{\alpha}{4}(D \exp (\psi(r))-\exp (-\psi(r)))\right] d r,
\end{gathered}
$$

where $\alpha=\mu D_{+}\left(C_{0} R_{g} T R_{p}^{2}\right)^{-1}, \psi_{s}=\psi(1, z), D=D_{-} / D_{+}$is the ratio of diffusion coefficients, and $\mu$ is the solution dynamic viscosity. The dependence of $\psi$ and $c_{v}$ on $z$ is not explicitly stated in the above formulas.

The boundary conditions for equations (8) are derived by putting $\psi(r, z)=0$ in (4) and taking into account the dimensional values of potential, ion concentration, and pressure in the reservoirs (see Fig. 1):

$$
\begin{array}{lll}
z=0: & p_{v}=-2 c_{0}, \quad c_{v}=c_{0}, & \phi_{v}=\varphi_{L} \\
z=1: & p_{v}=-2 c_{0}, \quad c_{v}=c_{0}, & \phi_{v}=\varphi_{R} .
\end{array}
$$

In this paper, we first propose a method for solving the problem (5)-(10). Note that the solution procedures for calculating the membrane potential at zero current in a nanopore with constant surface charge [19] or constant surface potential $[22,23]$ are essentially based on the fact that the salt concentrations in the reservoirs are different. They cannot be applied to the considered case, where the reservoir concentrations are equal, see (10).

First, it should be noted that the elements of matrix $L$ in (9) can be expressed as $L_{i j}=L_{i j}(z)$ provided that the dependencies $c_{v}=c_{v}(z)$ and $\phi_{v}=\phi_{v}(z)$ are known. Now let us integrate equations (8) with a variable upper limit from $z^{\prime}=0$ to $z^{\prime}=z$ taking into account boundary conditions (10):

$$
\begin{aligned}
& p_{v}(z)=-2 c_{0}+\int_{0}^{z}\left(L_{11} \bar{v}+L_{12} \bar{j}+L_{13} \bar{i}\right) d z^{\prime}, \\
& c_{v}(z)=c_{0} \exp \left(\int_{0}^{z}\left(L_{12} \bar{v}+L_{22} \bar{j}+L_{23} \bar{i}\right) d z^{\prime}\right), \\
& \phi_{v}(z)=\varphi_{L}+\int_{0}^{z}\left(L_{13} \bar{v}+L_{23} \bar{j}+L_{33} \bar{i}\right) d z^{\prime} .
\end{aligned}
$$

Setting $z=1$ in the above equations and taking into account (10) gives

$$
\begin{gathered}
\bar{v} \int_{0}^{1} L_{11} d z^{\prime}+\bar{j} \int_{0}^{1} L_{12} d z^{\prime}+\bar{i} \int_{0}^{1} L_{13} d z^{\prime}=0 \\
\bar{v} \int_{0}^{1} L_{12} d z^{\prime}+\bar{j} \int_{0}^{1} L_{22} d z^{\prime}+\bar{i} \int_{0}^{1} L_{23} d z^{\prime}=0 \\
\bar{v} \int_{0}^{1} L_{13} d z^{\prime}+\bar{j} \int_{0}^{1} L_{23} d z^{\prime}+\bar{i} \int_{0}^{1} L_{33} d z^{\prime}=\varphi_{R}-\varphi_{L} \\
-78-
\end{gathered}
$$


The numerical algorithm for solving the problem is formulated as follows:

Step 1. Introduce a mesh on $z$ direction with $n+1$ points $\left(z_{0}, z_{1}, \ldots, z_{n}\right)$, where $z_{0}=0$ and $z_{n}=1$.

Step 2. Set $c_{v}(z)=c_{0}, \phi_{v}(z)=\phi_{L}+\left(\phi_{R}-\phi_{L}\right) z$ as initial approximations.

Step 3. Solve problem (5)-(7) numerically for $c_{v}=c_{v}\left(z_{k}\right)$ and $\phi_{v}=\phi_{v}\left(z_{k}\right), k=0, \ldots, n$ and calculate the corresponding matrices $L_{i j}\left(z_{k}\right)$ according to $(9)$.

Step 4 . Determine the fluxes $\bar{v}, \bar{j}, \bar{i}$ by solving the linear system of equations (12).

Step 5. Calculate the solutions for $c_{v}(z)$ and $\phi_{v}(z)$ from (11) at $z=z_{k}, k=0, \ldots, n$.

Step 6. Repeat Steps 3-5 until the convergence for fluxes is achieved within the admissible error.

Step 7. Determine $p_{v}(z)$ from (11) and calculate the specific conductivity from (3).

\subsection{One-dimensional uniform potential model}

When the nanopore radius is comparable with or less than the Debye length, the profiles of potential, ion concentrations, and pressure in the radial direction become almost uniform. In this case, one can neglect the radial dependence of all quantities. This approach is known as Uniform potential (UP) model [19].

To describe the nanopore with electrically conductive surface within one-dimensional approach, we assume the presence of a thin dielectric Stern layer, which separates the pore wall and the diffuse layer (the pore interior), where ions and water molecules are located. In the Stern layer, there are no ions, and only water molecules can be present. The relation between the charge $\sigma$ induced on the diffuse layer boundary and the surface potential $\Phi_{s}$ is given by $[26,27]$

$$
\sigma=C_{s}\left(\Phi_{s}-\Phi\right) .
$$

Here $\Phi$ is the potential of the diffuse layer, which is radially uniform in the 1D model, and $C_{s}$ is the capacitance of the Stern layer. For a cylindrical nanopore, the latter is given by [27]

$$
C_{s}=\frac{\varepsilon_{s} \varepsilon_{0}}{\left(R_{p}-\delta_{s}\right) \ln \frac{R_{p}}{R_{p}-\delta_{s}}}
$$

where $\delta_{s}$ and $\varepsilon_{s}$ are the thickness and relative permittivity of the Stern layer, respectively. The dimensionless variables for the 1D model are introduced similarly to (1), where $R_{p}$ should be replaced by $R_{p}-\delta_{s}$.

The governing equations of UP model are derived from those of SC model taking into account essential simplification, which arises when the radial dependence of all quantities is ignored $[19,26,27]$ :

$$
\begin{gathered}
\bar{v}=\frac{1}{8 \alpha}\left(-\frac{d p}{d z}+X \frac{d \varphi}{d z}\right), \\
\bar{j}=c \bar{v}+\frac{1}{2}\left(-(D+1) \frac{d c}{d z}+(X(D+1)+c(D-1)) \frac{d \varphi}{d z}-(D-1) \frac{d X}{d z}\right) \\
\bar{i}=-X \bar{v}+\frac{1}{2}\left((D-1) \frac{d c}{d z}-(c(D+1)+X(D-1)) \frac{d \varphi}{d z}+(D+1) \frac{d X}{d z}\right) .
\end{gathered}
$$


Here $c=c_{+}+c_{-}$is the total concentration of cations and anions, and $X$ is the effective volume charge density, which is equal in magnitude and opposite in sing to the ionic charge density

$$
X=c_{-}-c_{+} \cdot
$$

The quantity $X$ is related to the surface charge density $\sigma$ at the pore wall by $X=2 \sigma\left(F\left(R_{p}-\delta\right)\right)^{-1}$. By defining the dimensionless capacitance $c_{s}=2 C_{s} R_{g} T\left(C_{*} F^{2}\left(R_{p}-\delta\right)\right)^{-1}$, we can write on the basis of (13)

$$
X=c_{s}\left(\varphi_{s}-\varphi\right) .
$$

Equations (15) can be solved with respect to the derivatives $d p / d z, d c / d z, d \varphi / d z$ to obtain a system of three ordinary differential equations. The boundary conditions inside the pore at the inlet from the left reservoir are written as

$$
p(0)=c(0)-2 c_{0}, \quad c(0)=\sqrt{X^{2}(0)+4 c_{0}^{2}}, \quad \phi(0)=\phi_{0} .
$$

Here, $\phi_{0}$ is the Donnan potential jump at the pore inlet. The corresponding concentrations and osmotic pressure jumps are described by first and second conditions in (17), respectively. Given that $c_{ \pm}(0)=c_{0} \exp \left(\mp\left(\phi_{0}-\phi_{L}\right)\right)$ and substituting these relations in (16), we obtain the equation to determine the potential $\phi_{0}$ :

$$
c_{s}\left(\varphi_{s}-\phi_{0}\right)=2 c_{0} \sinh \left(\phi_{0}-\phi_{L}\right) .
$$

The boundary conditions inside the pore at the outlet to the right reservoir have the form

$$
p(1)=c(1)-2 c_{0}, \quad c(1)=\sqrt{X^{2}(1)+4 c_{0}^{2}}, \quad \phi(1)=\phi_{1} .
$$

The relation analogues to (18) allows determination of potential $\phi_{1}$ :

$$
c_{s}\left(\varphi_{s}-\phi_{1}\right)=2 c_{0} \sinh \left(\phi_{1}-\phi_{R}\right) .
$$

Now the numerical algorithm for solving the problem can be formulated as:

Step 1. Set the initial approximations for fluxes $\bar{v}, \bar{j}, \bar{i}$.

Step 2. Determine the potentials $\phi_{0}$ and $\phi_{1}$ from (18) and (20) and set the initial conditions (17).

Step 3. Integrate equations (15) numerically from $z=0$ to $z=1$.

Step 4. Calculate the next approximation for fluxes from the condition of minimizing the residuals between the left-hand and right-hand parts of boundary conditions (19).

Step 5. Repeat Steps 3-4 until convergence for fluxes is achieved within the admissible error.

Step 6. Determine the specific conductivity from (3).

The integration is performed by the Runge-Kutta-Merson method of 5th order, while the boundary potentials and fluxes are determined by the one-dimensional and three-dimensional secant methods, respectively.

\section{Results and discussion}

We have investigated the ionic conductivity in an aqueous $\mathrm{KCl}$ solution for different surface potentials $\Phi_{s}$ and pore radii $R_{p}$. The fixed parameters in this study are electrolyte concentration $C_{0}=10 \mathrm{mM}$, ion diffusion coefficients $D_{+}=1.957 \cdot 10^{-9}$ and $D_{-}=2.032 \cdot 10^{-9} \mathrm{~m}^{2} / \mathrm{s}$, pore 
length $L_{p}=100 \mu \mathrm{m}$, reservoir potentials $\Phi_{L}=-0.025 \mathrm{~V}$ and $\Phi_{R}=0.025 \mathrm{~V}$, relative permittivity of water $\varepsilon=78.49$, viscosity $\mu=0.88 \cdot 10^{-3} \mathrm{~Pa} \cdot \mathrm{s}$, and temperature $T=298.15 \mathrm{~K}$. The Stern layer thickness is $\delta_{s}=0.5 \mathrm{~nm}$, and the Stern layer permittivity is taken as that of water $\varepsilon_{s}=78.49$.

Let us first analyze the profiles of potential, ion concentrations, and pressure along the nanopore. Fig. 2 shows the comparison between $1 \mathrm{D}$ and $2 \mathrm{D}$ models for a nanopore with the radius $R_{p}=2 \mathrm{~nm}$ and surface potential $\Phi_{s}=0.025 \mathrm{~V}$. For the $2 \mathrm{D}$ model, the cross-sectionally averaged values are shown, see formula (2). The Donnan potential jump, ion concentration jumps, and osmotic pressure jump are seen at the interface between the nanopore and both reservoirs. The surface potential is positive, so the concentration of cations (anions) inside the nanopore is lower (higher) than in the bulk. The 1D and 2D models provide close results for potential and cation concentration profiles, while the variation of pressure and anion concentration along the pore are slightly higher in the $1 \mathrm{D}$ model. Note that the Debye length for $C_{0}=10 \mathrm{mM}$ is $3.04 \mathrm{~nm}$, which is larger than the pore radius of $2 \mathrm{~nm}$.

Fig. 3 shows the profiles of all quantities in the nanopore of $10 \mathrm{~nm}$ radius. Here the 1D model leads to overestimated values of potential, pressure, and anion concentration, while the cation concentration is underestimated in comparison with the 2D model. In this case, the Debye length $(3.04 \mathrm{~nm})$ is smaller than the nanopore radius $(10 \mathrm{~nm})$. Thus, the decrease of potential and the corresponding radial variations of cation/anion concentrations in the 2D model lead in the noticeable deviation of cross-sectionally averaged values from the 1D model.
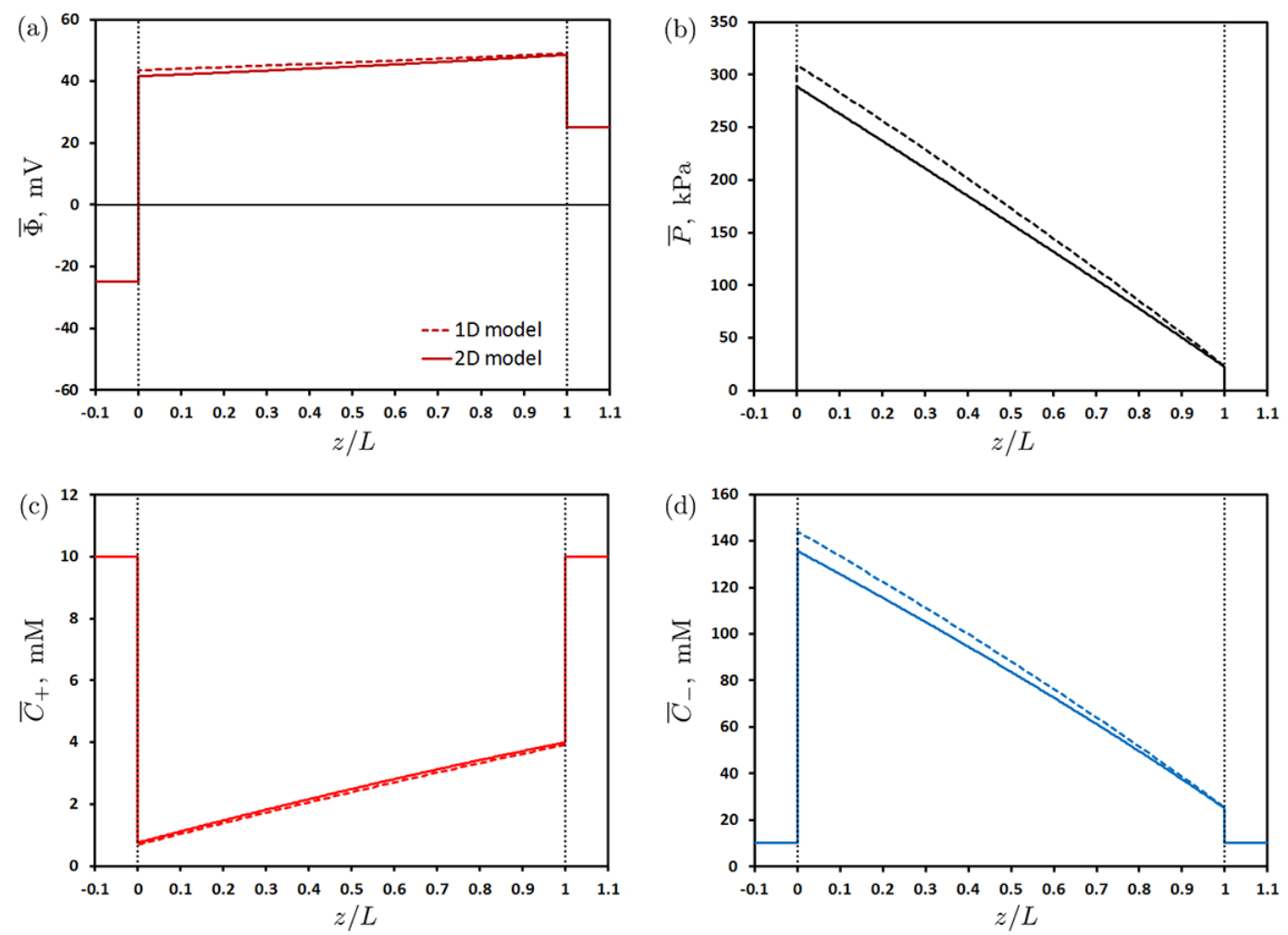

Fig. 2. The profiles of cross-sectionally averaged potential (a), pressure (b), concentrations of cations (c) and anions (d) along the nanopore with the radius $R_{p}=2 \mathrm{~nm}$ and surface potential $\Phi_{s}=0.025 \mathrm{~V}$ 

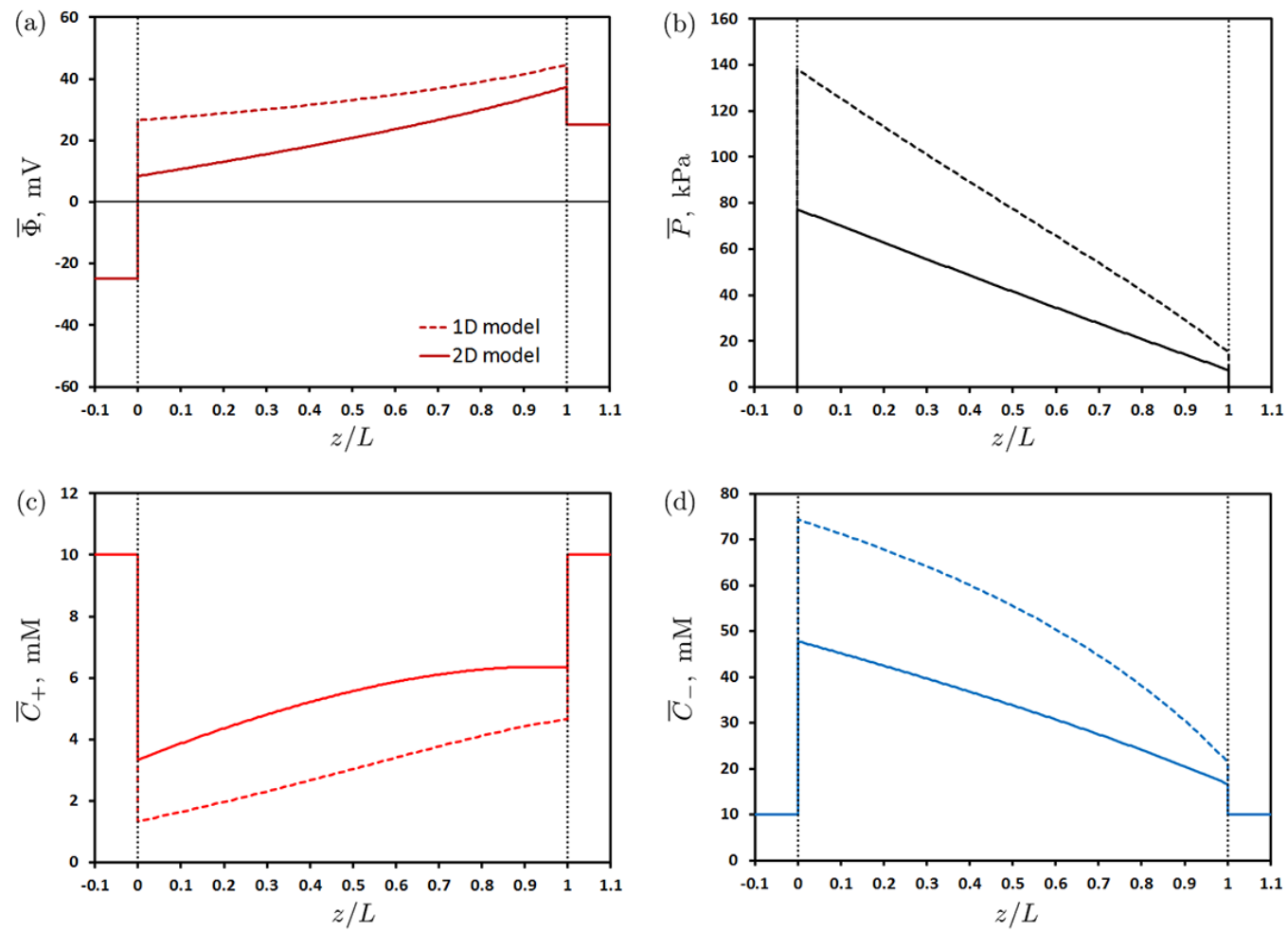

Fig. 3. The profiles of cross-sectionally averaged potential (a), pressure (b), concentrations of cations (c) and anions (d) along the nanopore with the radius $R_{p}=10 \mathrm{~nm}$ and surface potential $\Phi_{s}=0.025 \mathrm{~V}$

The conductivity dependence on the applied surface potential for nanopores of 2 and $10 \mathrm{~nm}$ radius is shown in Fig. 4. The increase of surface potential magnitude leads to the enhancement of conductivity. It occurs due to the increase of counter-ion concentration inside the nanopore and, consequently, the ionic current. The 1D and 2D models provide very close results since the Debye length $(3.04 \mathrm{~nm})$ is higher than the pore radius $(2 \mathrm{~nm})$. Nevertheless, the 1D model gives slightly higher conductivity values (Fig. 4 a) due to higher concentration of counter-ions inside the nanopore in comparison with the $2 \mathrm{D}$ model, see Fig. $2 \mathrm{~d}$. For a nanopore with the radius of $10 \mathrm{~nm}$ (Fig. $4 \mathrm{~b}$ ), the pore radius exceeds the Debye length, and cross-sectionally averaged ion concentrations inside the nanopore in the $2 \mathrm{D}$ model deviate from the bulk reservoir values much smaller in comparison with the 1D model, see Fig. $3 \mathrm{c}, \mathrm{d}$. Thus, the $2 \mathrm{D}$ model provides much lower magnitudes of ionic conductivity except the region near $\Phi_{s}=0$, where the nanopore is not charged. However, the qualitative dependence of conductivity on the applied potential is similar in both models.

The dependence of ionic conductivity on the nanopore radius for fixed surface potential $\Phi_{s}=0.025 \mathrm{~V}$ is presented in Fig. 5. According to the $2 \mathrm{D}$ model, the conductivity decreases with the nanopore radius due to lowering of counter-ion concentration inside the nanopore, which is in turn caused by the decreasing Debye length. However, the 1D model first shows some decrease and then the increase of conductivity when the nanopore radius becomes larger, which is physically incorrect. Nevertheless, the $1 \mathrm{D}$ and 2D models provide close results when the nanopore radius is smaller than the Debye length. The discrepancy between them grows with increasing the nanopore radius. 

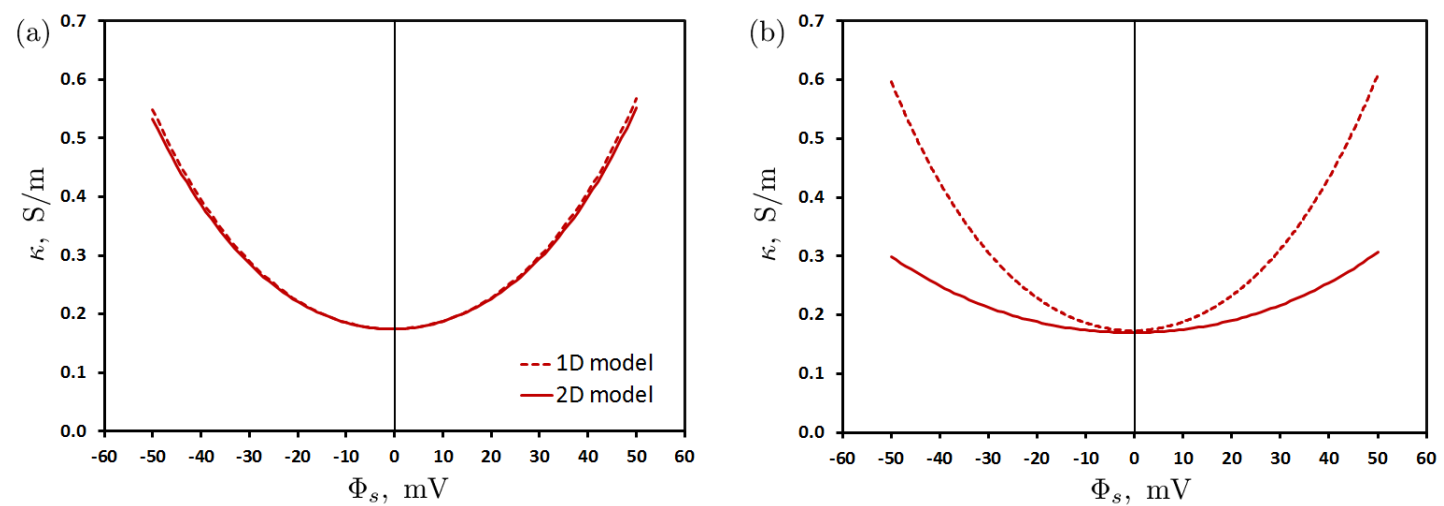

Fig. 4. The dependence of ionic conductivity on the surface potential for a nanopore with the radius $R_{p}=2 \mathrm{~nm}$ (a) and $R_{p}=10 \mathrm{~nm}(\mathrm{~b})$

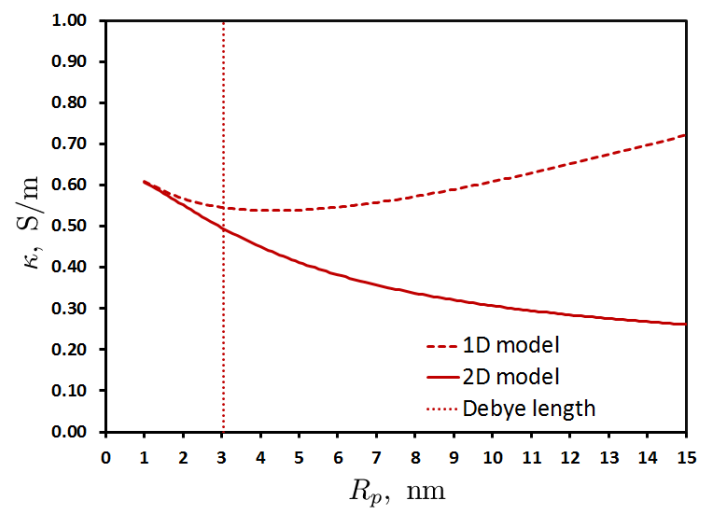

Fig. 5. The dependence of ionic conductivity on the nanopore radius for surface potential $\Phi_{s}=0.025 \mathrm{~V}$

\section{Conclusion}

In this work, we have investigated the ionic conductivity of a single nanopore with electrically conductive surface on the basis of two-dimensional (2D) Space charge model and one-dimensional (1D) Uniform potential model. The models are derived from the Navier-Stokes, Nernst-Planck, and Poisson equations. The generalization of Space charge model to calculating the ion transport under the applied potential difference in a nanopore with constant surface potential is proposed for the first time. The numerical algorithms for solving the model equations are suggested. It is shown that the ionic conductivity becomes larger with increasing the magnitude of applied surface potential due to the increase of counter-ion concentration inside the nanopore. A good agreement is found between the $1 \mathrm{D}$ and $2 \mathrm{D}$ models when the pore radius is smaller than or comparable with the Debye length. Otherwise, the 1D model essentially overestimates the ionic conductivity. According to the 2D model, the ionic conductivity decreases with increasing the nanopore radius, while the 1D model predicts the opposite trend, which is not physically correct. 
The reported study was funded by Russian Foundation for Basic Research, Government of Krasnoyarsk Territory, Krasnoyarsk Regional Fund of Science, to the research project 1848-242011 "Mathematical modelling of synthesis and ionic transport properties of conductive nanoporous membranes".

A. I. Krom is thankful for the support provided by the Krasnoyarsk Mathematical Center and financed by the Ministry of Science and Higher Education of the Russian Federation in the framework of the establishment and development of regional Centers for Mathematics Research and Education (Agreement no. 075-02-2020-1631).

\section{References}

[1] H.Strathmann, Ion-exchange membrane separation processes, Elsevier, Amsterdam-Boston, 2004.

[2] A.Figoli, J.Hoinkis, S.A.Altinkaya, J.Bundschuh, Application of nanotechnology in membranes for water treatment, CRC Press, 2017.

[3] A.Cipollina, G.Micale, Sustainable energy from salinity gradients, Elsevier/Woodhead Publishing, 2016.

[4] F.G.Bănică, Chemical sensors and biosensors: fundamentals and applications, John Wiley \& Sons, Chichester, UK, 2012.

[5] W.Sparreboom, A.van den Berg, J.C.T. Eijkel, Principles and applications of nanofluidic transport, Nature nanotech., 4(2009), 713-720. DOI: 10.1038/nnano.2009.332

[6] Z.S.Siwy, S.Howorka, Engineered voltage-responsive nanopores, Chem. Soc. Rev., 39(2010), 1115-1132. DOI: 10.1039/b909105j

[7] X.Hou, W.Guo, L.Jiang, Biomimetic smart nanopores and nanochannels, Chem. Soc. Rev., 40(2011), 2385-2401. DOI: 10.1039/C0CS00053A

[8] H.Strathmann, Electrodialysis, a mature technology with a multitude of new applications, Desalination, 264(2010), 268-288. DOI: 10.1016/j.desal.2010.04.069

[9] S.Porada, R.Zhao, A. van der Wal, V.Presser, P.M.Biesheuvel, Review on the science and technology of water desalination by capacitive deionization, Progress in Mater. Sci., 58(2013), 1388-1442. DOI: /10.1016/j.pmatsci.2013.03.005

[10] L.Zhang, S.R.Chae, Z.Hendren, J.S.Park, M.R.Wiesner, Recent advances in proton exchange membranes for fuel cell applications, Chem. Eng. J., 204-206(2012), 87-97.

DOI: $10.1016 /$ j.cej.2012.07.103

[11] G.B.Westermann-Clark, J.L.Anderson, Experimental verification of the Space-Charge model for electrokinetics in charged microporous membranes, J. Electrochem. Soc., 130(1983), 839-847. DOI: 10.1149/1.2119832

[12] R.Lteif, L.Dammak, C.Larchet, B.Auclair, Conductivité électrique membranaire: étude de l'effet de la concentration, de la nature de l'électrolyte et de la structure membranaire, Europ. Polymer J., 35(1999), 1187-1195. DOI: 10.1016/S0014-3057(98)00213-4 
[13] K.Yazda, S.Tahir, T.Michel, B.Loubet, M.Manghi, J.Bentin, F.Picaud, J.Palmeri, F.Henn, V.Jourdain, Voltage-activated transport of ions through single-walled carbon nanotubes, Nanoscale, 9(2017), 11976-11986. DOI: 10.1039/C7NR02976D

[14] C.R.Martin, M.Nishizawa, K.Jirage, M.Kang, S.B.Lee, Controlling ion-transport selectivity in gold nanotubule membranes, Adv. Mater., 13(2001), 1351-1362.

DOI: 10.1002/1521-4095(200109)13:18\%3C1351::AID-ADMA1351\%3E3.0.CO;2-W

[15] P.Gao, C.R.Martin, Voltage charging enhances ionic conductivity in gold nanotube membranes, ACS Nano, 8(2014), 8266-8272. DOI: $10.1021 / \mathrm{nn} 502642 \mathrm{~m}$

[16] D.V.Lebedev, V.S.Solodovnichenko, M.M.Simunin, I.I.Ryzhkov. Effect of electric field on ion transport in nanoporous membranes with conductive surface, Petrol. Chem., 58(2018), no. $6,474-481$. DOI: $10.1134 /$ S0965544118060075

[17] R.J.Gross, J.F.Osterle, Membrane transport characteristics of ultrafine capillaries, J. Chem. Phys., 49(1968), 228-234. DOI: 10.1063/1.1669814

[18] A.Szymczyk, P.Fievet, B.Aoubiza, C.Simon, J.Pagetti, An application of the space charge model to the electrolyte conductivity inside a charged microporous membrane, J. Membr. Sci., 161(1999), 275-285. DOI: 10.1016/S0376-7388(99)00118-0

[19] P.B.Peters, R.van Roij, M.Z.Bazant, P.M.Biesheuvel, Analysis of electrolyte transport through charged nanopores, Phys. Rev. E, 93(2016), 053108.

DOI: 10.1103/PhysRevE.93.053108

[20] P.M.Biesheuvel, M.Z.Bazant, Analysis of ionic conductance of carbon nanotubes, Phys. Rev. E, 94(2016), 050601. DOI: 10.1103/PhysRevE.94.050601

[21] M.Z.Bazant, T.M.Squires, Induced-charge electrokinetic phenomena, Curr. Op. Coll. Inter. Sci., 15(2010), 203-213. DOI: 10.1016/j.cocis.2010.01.003

[22] I.I.Ryzhkov, D.V.Lebedev, V.S.Solodovnichenko, A.V.Shiverskiy, M.M.Simunin, Inducedcharge enhancement of the diffusion potential in membranes with polarizable nanopores, Phys. Rev. Lett., 119(2017), 226001. DOI: 10.1103/PhysRevLett.119.226001

[23] I.I.Ryzhkov, D.V.Lebedev, V.S.Solodovnichenko, A.V.Minakov, M.M.Simunin, On the origin of membrane potential in membranes with polarizable nanopores, J. Membrane Science, 549(2018), 616-630. DOI: 10.1016/j.memsci.2017.11.073

[24] I.I.Ryzhkov, A.S.Vyatkin, A.V.Minakov. Theoretical study of electrolyte diffusion through polarizable nanopores, J. Siber. Fed. Univer.: Math. Phys., 11(2018), no. 4, 494-504.

DOI: 10.17516/1997-1397-2018-11-4-494-504

[25] I.I.Ryzhkov, A.S.Vyatkin, M.I.Medvedeva, Modelling of electrochemically switchable ion transport in nanoporous membranes with conductive surface, J. Siber. Fed. Univer.: Math. Phys., 12(2019), no. 5, 579-589.

DOI: $10.17516 / 1997-1397-2019-12-5-579-589$

[26] L.Zhang, P.M.Biesheuvel, I.I.Ryzhkov, Theory of ion and water transport in electronconducting membrane pores with $\mathrm{pH}$-dependent chemical charge, Physical Review Applied, 12(2019), 014039. DOI: 10.1103/PhysRevApplied.12.014039 
[27] I.I.Ryzhkov, A.S.Vyatkin, E.V.Mikhlina, Modelling of conductive nanoporous membranes with switchable ionic selectivity, Membranes and Membrane Technologies, 2(2020), 10-19. DOI: $10.1134 /$ S2517751620010072

\title{
Моделирование ионной проводимости нанопор с проводящей поверхностью
}

Артур И. Кром

Институт вычислительного моделирования СО РАН Красноярск, Российская Федерация

Мария И. Медведева

Сибирский федеральный университет Красноярск, Российская Федерация

Илья И. Рыжков

Институт вычислительного моделирования СО РАН

Красноярск, Российская Федерация Сибирский федеральный университет

Красноярск, Российская Федерация

\begin{abstract}
Аннотация. В работе теоретически исследуется ионная проводимость нанопоры с электропроводящей поверхностью. Для описания ионного переноса под действием приложенной разности потенциалов в нанопоре с постоянным поверхностным потенциалом впервые предложено обобщение двумерной модели пространственного заряда на основе уравнений Навье-Стокса, Нернста-Планка и Пуассона. Результаты расчетов сравниваются с данными, полученными на основе одномерной модели равномерного потенциала, которая выводится из модели пространственного заряда в предположении независимости потенциала, концентраций ионов и давления от радиальной координаты. Установлено, что увеличение абсолютной величины потенциала поверхности приводит к росту проводимости в силу повышения концентрации противоионов внутри нанопоры. Показано, что обе модели дают близкие значения проводимости для нанопор, радиус которых меньше длины Дебая. Для нанопор большего радиуса одномерная модель дает завышенные значения проводимости. Согласно двумерной модели, ионная проводимость уменьшается с увеличением радиуса нанопоры, что не подтверждается одномерной моделью, которая даёт физически некорректный результат в этом случае.
\end{abstract}

Ключевые слова: нанопора, ионная проводимость, модель пространственного заряда, модель однородного потенциала. 